\title{
NONLINEAR DENSITY-DEPENDENT DIFFUSION FOR COMPETING SPECIES INTERACTIONS: LARGE-TIME ASYMPTOTIC BEHAVIOUR
}

\author{
by ANTHONY W. LEUNG
}

(Received 2nd August 1983)

\section{Introduction}

In many biological diffusion-reaction studies, it was found that one should include the effect of density dependent rates, drift terms and spatially varying growth rates, in order to obtain more accurate results. (See e.g. [7], [10], [8], [3]). On the other hand, many recent mathematical results on reaction-diffusion systems do not include such general setting. This article investigates the behaviour of competing-species reaction-diffusion model under this more general situation. Efforts are made to obtain results concerning coexistence, survival and extinction, by methods similar to that in [5], [6].

We determine the nature of nonlinear density-dependent diffusion, which would still allow results analogous to those in [6]. (Note the positivity and monotonic nondecreasing property of $\sigma_{i}(s)$ in (1.5) below). Conditions for existence of positive coexistence steady states were found in [5], [6], [12]. They were of the nature that growth rates of the species are uniformly larger than certain positive constants related to the first eigenvalue. In the case of highly heterogeneous environment, such conditions are difficult to satisfy in reality. In Theorem 2.1, we determine a sufficient condition for survival, in terms of only regionally large enough growth rates (see (2.2b)). Theorem 2.3 generalizes Theorem 2.1 to the case of several coexistence species. Theorem 2.2 establishes a-priori bounds for the solutions by means of nonsmooth lower and upper solutions. It extends some results given in [9] (see remarks before Theorem 2.2), and it is used to prove all the other theorems in this article. Theorem 3.1 shows that results concerning extinction are similar to that in [6], provided that the rate of change of diffusion rate with respect to density is smaller than a certain constant. This constant is related to the principle eigenfunction of a larger domain. Theorem 4.1 shows the existence of solutions to the initial-boundary value problem, completing the validity of our model for the actual problem. The existence theorem for quasilinear systems in [4] presumes at most quadratic growth conditions of reaction terms in its dependence on density. With the aid of a variation of Theorem 2.1, Theorem 4.1 shows that this assumption can be removed. Moreover, bounds for the solutions are also found.

We now clarify our notations and equations. Let $l, 0<l<1$ be a fixed number. We consider an open connected bounded set $\mathscr{D}$ in $\mathbb{R}^{m}, m \geqq 2$, with $\overline{\mathscr{D}}$ denoting its closure. $H^{2+l}(\overline{\mathscr{D}})$ denotes the Banach space of all real-valued functions with all first and second derivatives continuous in $\mathscr{D}$, and with finite value for the norm $|\cdot|_{\mathscr{D}}^{(2+l)}$ (as described in [4], p. 7). The boundary of $\mathscr{D}$, denoted by $\delta \mathscr{D}$, will belong to class $H^{2+\ell}$ ([4], p. 9). For 
any $T>0$, let $\mathscr{D}_{T}=\mathscr{D} \times(0, T) . H^{2+l, 1+l / 2}\left(\overline{\mathscr{D}}_{T}\right)$ denotes the Banach space of all realvalued functions having all derivatives of the form $D^{\alpha} D_{t}^{r}, 2 r+|\alpha| \leqq 2$, continuous in $\overline{\mathscr{D}}_{T}$ and having finite norm $|\cdot|_{\mathscr{D}_{T}}^{(2+l)}$ (as described in [4]). For vector functions in $H^{2+l}(\overline{\mathscr{D}})$ or $H^{2+l, 1+l / 2}\left(\mathscr{D}_{T}\right)$, we mean that all its components belong to the corresponding space. For a vector $v=\left(v_{1}, \ldots, v_{n}\right),|v|=\left(\sum_{i=1}^{n} v_{i}^{2}\right)^{1 / 2} . \quad \nabla=\left(\left(\partial / \partial x_{1}\right), \ldots,\left(\partial / \partial x_{m}\right)\right)$ denotes the gradient, div $v=\sum_{i=1}^{n}\left(\partial v_{i} / \partial x_{i}\right)$ denotes the divergence, and $\Delta=\sum_{i=1}^{n}\left(\partial^{2} / \partial x_{i}^{2}\right)$ denotes the Laplacian.

In our equations below, we assume that diffusivity depends on concentration, giving rise to the term $\operatorname{div}\left(\sigma_{i}\left(u_{i}\right) \nabla u_{i}\right)$, with $\sigma_{i}\left(u_{i}\right)$ expressing the concentration dependence (cf. $[10]$, [7]). Moreover, the intrinsic growth rates will be assumed as functions of position $x=\left(x_{1}, \ldots, x_{m}\right)$. We consider the following initial Dirichlet boundary value problem for $n$ competing-species reaction-diffusion.

$$
\begin{gathered}
\frac{\partial u_{i}}{\partial t}=\operatorname{div}\left(\sigma_{i}\left(u_{i}\right) \nabla u_{i}\right)+u_{i}\left[a_{i}(x)+f_{i}\left(u_{1}, \ldots, u_{n}\right)\right] \\
\text { for }(x, t)=\left(x_{1}, \ldots, x_{m}, t\right) \in \mathscr{D} \times(0, T], T>0, i=1, \ldots, n \\
u_{i}(x, 0)=\phi_{i}(x), x \in \overline{\mathscr{D}} ; u_{i}(x, t)=\Phi_{i}(x),(x, t) \in \delta \mathscr{D} \times[0, T]
\end{gathered}
$$

The functions $f_{i}: \mathbb{R}^{n} \rightarrow \mathbb{R}$ have Hölder continuous partial derivatives up to second order in compact sets, $i=1, \ldots, n$. (The smoothness and compatibility of $\phi_{i}, \Phi_{i}$ will be prescribed later). The functions, $f_{i}$, describing competing interactions satisfy:

$$
\frac{\partial f_{i}}{\partial u_{j}}<0, i, j=1, \ldots, n \text { in }\left\{\left(u_{1}, \ldots, u_{n}\right) \mid u_{i} \geqq 0, i=1, \ldots, n\right\}, f_{i}(0,0)=0
$$

and

$$
\sup _{s \geqq 0} \frac{\partial f_{i}}{\partial u_{i}}(0, \ldots, 0, s, 0, \ldots, 0) \stackrel{(\text { def })}{=} r_{i}<0
$$

where $s \geqq 0$ occurs at the $i$ th component, $i=1, \ldots, n$.

The intrinsic growth rate function of the $i$ th species, $a_{i}(x)$, is in $H^{1+l}(\overline{\mathscr{D}})$ and

$$
a_{i}(x) \geqq 0 \text { in } \overline{\mathscr{D}}, \quad i=1, \ldots, n .
$$

The diffusivity functions $\sigma_{i}(s)$ satisfy:

$$
\sigma_{i}(0)>0, \sigma_{i}(s) \text { in } H^{\alpha}(R), \sigma_{i}^{\prime}(s) \geqq 0 \text { in }[0, \infty), \sigma_{i}^{\prime \prime}(s) \text { is continuous in }[0, \infty), i=1, \ldots, n
$$

These assumptions and equations are biologically plausible, and include the more general properties of diffusion-reaction described above. The smoothness and other assumptions are made convenient enough so that excessive technicalities do not arise. 


\section{A-priori and positive lower bounds, criteria for survival and coexistence}

The initial-boundary value problem (1.1), under conditions (1.2) to (1.5) and appropriate smoothness conditions for the initial boundary function, would possess a solution in $H^{2+1,1+1 / 2}\left(\overline{\mathscr{D}}_{T}\right)$, each $T>0$. The detailed proof for existence is delayed to Section 4 , because we will need the method of establishing a-priori bound for solutions given in Theorem 2.2.

The hypotheses in Theorems 2.1 and 2.3 are motivated by biological intuition. We assume that the intrinsic growth rate, $a_{k}(x)$. of a particular $k$ th species is locally high in a subdomain $\mathscr{D}^{\prime}$ of $\mathscr{D}$. We will obtain a criteria on $a_{k}(x)$ which ensures that the population $u_{k}(x, t)$ will be bounded below by a positive constant in compact subsets of $\mathscr{D}^{\prime}$ for all $t$. Such criteria can thus be interpreted as a survival condition for the $k$ th species. Comparing with results in [5], [6], condition (2.2b) below is much more realistic, because the growth rate here does not have to be "large" on the entire domain. One only needs locally high growth rates to sustain survival.

Theorem 2.1. Let $k$ be an integer, $1 \leqq k \leqq n$. Let $u=\left(u_{1}, \ldots, u_{n}\right)$ be a solution of $(1.1)$ in the class $H^{2+1,1+l / 2}\left(\overline{\mathscr{D}}_{T}\right), T>0$, initially satisfying:

$$
0 \leqq u_{i}(x, 0) \leqq b_{i}, x \in \overline{\mathscr{D}}, i=1, \ldots, n
$$

where $b_{i}$ are positive numbers satisfying $b_{i} \geqq\left|r_{i}^{-1}\right| \cdot \max \left\{a_{i}(x) \mid x \in \overline{\mathscr{D}}\right\}$. Suppose that there exists a subdomain $\mathscr{D}^{\prime} \subseteq \mathscr{D}$ (with principle eigenvalue $\lambda^{\prime}$ i.e. $\lambda=\lambda^{\prime}>0$ is the first eigenvalue for the problem $\Delta \phi+\lambda \phi=0$ in $\mathscr{D}^{\prime}, \phi=0$ on $\left.\delta \mathscr{D}^{\prime}\right)$ with the properties:

$$
\begin{gathered}
0<u_{k}(x, 0), x \in \overline{\mathscr{D}}^{\prime} ; \\
a_{k}(x)-\sigma_{k}(0) \lambda^{\prime}+f_{k}\left(b_{1}, \ldots, b_{k-1}, 0, b_{k+1}, \ldots, b_{n}\right)>0
\end{gathered}
$$

for all $x \in \overline{\mathscr{D}}^{\prime}$. Then the solution $u$ satisfies:

$$
0<u_{k}(x, t) \text { for }(x, t) \in \mathscr{D}^{\prime} \times[0, T] .
$$

Moreover, $u_{k}(x, t) \geqq \delta>0$ for all $x$ in any compact set contained in $\mathscr{D}^{\prime}, 0 \leqq t \leqq T$ (where $\delta$ is some constant depending on the compact set, independent of $T$ ); and

$$
0 \leqq u_{i}(x, t) \leqq b_{i} \quad \text { for } \quad(x, t) \in \overline{\mathscr{D}}_{T}, \quad i=1, \ldots, n
$$

Proof. We shall construct lower and upper solutions $v_{i}, w_{i}$ satisfying differential inequalities (2.9), (2.10), with $v_{i}, w_{i}$ replacing $\alpha_{i}, \beta_{i}$ respectively. Then, we apply Theorem 2.2 below to conclude $u_{k}(x, t) \geqq v_{k}(x, t)$ in $\bar{D}_{T}$. The function $v_{k}$ will be positive for $x$ in the interior of $\mathscr{D}^{\prime}$, thus implying the survival of the $k$ th species. Let $\theta(x)$ be a positive eigenfunction in $\mathscr{D}^{\prime}$, associated with the principle eigenvalue $\lambda=\lambda^{\prime}$. Define $v_{i}(x, t) \equiv 0$ in $\overline{\mathscr{D}}_{T}$ for $i \neq k, 1 \leqq i \leqq n ;$ and

$$
v_{k}(x, t)= \begin{cases}\varepsilon \theta(x), & \text { if } x \in \mathscr{D}^{\prime} \\ 0, & \text { if } x \in \overline{\mathscr{D}} \backslash \mathscr{D}^{\prime}\end{cases}
$$


in $\overline{\mathscr{D}}_{T}$. Here $\varepsilon$ is a sufficiently small positive constant to be determined later. For $i=1, \ldots, n$, define $w_{i}(x, t) \equiv b_{i}$ in $\mathrm{n} \overline{\mathscr{D}}_{T}$. We have the following inequality, for $i=1, \ldots, n$ :

$$
\begin{aligned}
& \operatorname{div}\left(\sigma_{i}\left(w_{i}\right) \nabla w_{i}\right)+w_{i}\left[a_{i}(x)+f_{i}\left(v_{1}, \ldots, v_{i-1}, w_{i}, v_{i+1}, \ldots v_{n}\right)\right]-\frac{\partial w_{i}}{\partial t} \\
& \quad \leqq w_{i}\left[a_{i}(x)+f_{i}\left(0, \ldots, 0, w_{i}, 0, \ldots, 0\right)\right] \\
& \quad=w_{i}\left[a_{i}(x)+\int_{0}^{w_{i}} \frac{\partial f_{i}}{\partial u_{i}}\left(0, \ldots, 0, u_{i}, 0, \ldots, 0\right) d u_{i}\right] \\
& \quad \leqq w_{i}\left[a_{i}(x)+r_{i} w_{i}\right] \leqq b_{i}\left[a_{i}(x)-\max \left\{a_{i}(x) \mid x \in \overline{\mathscr{D}}\right\}\right] \leqq 0
\end{aligned}
$$

for $(x, t) \in \mathscr{D} \times[0, T]$. For $i \neq k$, clearly we have

$$
\operatorname{div}\left(\sigma_{i}\left(v_{i}\right) \nabla v_{i}\right)+v_{i}\left[a_{i}(x)+f_{i}\left(w_{1}, \ldots, w_{i-1}, v_{i}, w_{i+1}, \ldots, w_{n}\right)\right]-\frac{\partial v_{i}}{\partial t}=0
$$

for $(x, t) \in \mathscr{D} \times[0, T]$. For $i=k,(2.7)$ is clearly valid for $(x, t) \in\left(\mathscr{D} \backslash \overline{\mathscr{D}}^{\prime}\right) \times[0, T]$. If $(x, t) \in \mathscr{D}^{\prime} \times[0, T]$, we have

$$
\begin{aligned}
\operatorname{div} & \left(\sigma_{k}\left(v_{k}\right) \nabla v_{k}\right)+v_{k}\left[a_{k}(x)+f_{k}\left(w_{1}, \ldots, w_{k-1}, v_{k}, w_{k+1}, \ldots, w_{n}\right)\right]-\frac{\partial v_{k}}{\partial t} \\
= & \sigma_{k}\left(v_{k}\right) \Delta v_{k}+\sigma_{k}^{\prime}\left(v_{k}\right)\left|\operatorname{grad} v_{k}\right|^{2}+\varepsilon \theta(x)\left[a_{k}(x)+f_{k}\left(w_{1}, \ldots, v_{k}, \ldots, w_{n}\right)\right] \\
= & \varepsilon \theta(x)\left[a_{k}(x)-\sigma_{k}(\varepsilon \theta(x)) \lambda^{\prime}+f_{k}\left(b_{1}, \ldots, b_{k-1}, \varepsilon \theta, b_{k+1}, \ldots, b_{n}\right)\right]+\sigma_{k}^{\prime}(\varepsilon \theta)\left|\operatorname{grad} v_{k}\right|^{2}
\end{aligned}
$$

Now, choose $\varepsilon>0$ sufficiently small so that the expression in (2.8) is positive in $\mathscr{D}^{\prime} \times[0, T]$. (This is possible due to hypotheses $(1.5)$ and $\left.(2.1)\right)$. Let $\left(u_{1}, \ldots, u_{n}\right)$ be a solution of (1.1) satisfying (2.2) as stated. Reduce the choice of $\varepsilon>0$, if necessary, so that $u_{k}(x, 0)>v_{k}(x, 0)=\varepsilon \theta(x)$ for $x \in \bar{D}^{\prime}$ (note that this will not affect the sign of the expression in (2.8)). Utilizing inequalities (2.6) to (2.8) and Theorem 2.2 below, we conclude that

$$
0 \leqq v_{i} \leqq u_{i}(x, t) \leqq w_{i}=b_{i}, i=1, \ldots, n
$$

for $(x, t) \in \overline{\mathscr{D}} \times[0, T]$. From the definition of $v_{k}$ in (2.5), we have (2.3) and the strict positivity of $u_{k}$ in compact subsets of $\mathscr{D}^{\prime}$ as stated in the theorem.

Remark. The following theorem is a comparison result similar to that found in [2]. As in [2], nonsmooth comparison functions are used. However, the differential operator here, $\operatorname{div}\left(\sigma_{i}\left(u_{i}\right) \nabla u_{i}\right)$, has its coefficients $\sigma_{i}\left(u_{i}\right)$ depending on $u_{i}$; and for $x \in \delta \mathscr{D}^{\prime}$, our lower solution $v_{k}$ is not the maximum of a finite collection of regular subsolution in a neighbourhood of the point. Consequently, results in [2] need to be extended here for our purpose. 
Theorem 2.2. Let $\mathscr{D}^{\prime} \subseteq \mathscr{D}$ be a subdomain, with $\lambda^{\prime}$ as its principle eigenvalue and $\theta(x)$ a positive eigenfunction in $\mathscr{D}^{\prime}$. Let $j$ be an integer $1 \leqq j \leqq n ; \alpha_{i}(x, t) \leqq 0$ in $\overline{\mathscr{D}}_{T}$ if $i \neq j, 1 \leqq i \leqq n$, and

$$
\alpha_{j}(x, t)= \begin{cases}\delta \theta(x) & \text { if }(x, t) \in \mathscr{D}^{\prime} \times[0, T] \\ 0 & \text { if }(x, t) \in\left(\overline{\mathscr{D}} \backslash \mathscr{D}^{\prime}\right) \times[0, T]\end{cases}
$$

where $\delta>0$ is a. constant. Let $\beta_{i}(x, t)$ be nonnegative functions in $H^{2+l, 1+l / 2}\left(\overline{\mathscr{D}}_{T}\right)$ for $i=1, \ldots, n$. Suppose that $\alpha_{i}, \beta_{i}$ satisfy:

$$
\begin{gathered}
\alpha_{i}(x, t) \leqq \beta_{i}(x, t) \text { for }(x, t) \in \overline{\mathscr{D}}_{T} ; \\
\operatorname{div}\left(\sigma_{i}\left(\alpha_{i}\right) \nabla \alpha_{i}\right)+\alpha_{i}\left[a_{i}(x)+f_{i}\left(\beta_{1}, \ldots, \beta_{i-1}, \alpha_{i}, \beta_{i+1}, \ldots, \beta_{n}\right)\right]-\frac{\partial \alpha_{i}}{\partial t} \geqq 0 \\
\operatorname{div}\left(\sigma_{i}\left(\beta_{i}\right) \nabla \beta_{i}\right)+\beta_{i}\left[a_{i}(x)+f_{i}\left(\alpha_{1}, \ldots, \alpha_{i-1}, \beta_{i}, \alpha_{i+1}, \ldots, \alpha_{n}\right)\right]-\frac{\partial \beta_{i}}{\partial t} \leqq 0
\end{gathered}
$$

for $(x, t) \in \mathscr{D} \times(0, T], \quad i=1, \ldots, n, \quad$ except for $i=j$ in (2.9) valid only for $(x, t) \in\left(\mathscr{D} \backslash \delta \mathscr{D}^{\prime}\right) \times\left(0,{ }^{T}\right)$. Let $\left(u_{1}, \ldots, u_{n}\right), \quad u_{i} \in H^{2+l .1+l / 2}\left(\overline{\mathscr{D}}_{T}\right)$, be a solution of the differential equation in (1.1) with initial boundary conditions satisfying:

$$
\begin{gathered}
\alpha_{i}(x, 0) \leqq u_{i}(x, 0) \leqq \beta_{i}(x, 0), x \in \overline{\mathscr{D}} \\
\alpha_{i}(x, t) \leqq u_{i}(x, t) \leqq \beta_{i}(x, t),(x, t) \in \delta \mathscr{D} \times[0, T]
\end{gathered}
$$

for $i=1, \ldots, n$. Then we have

$$
\alpha_{i}(x, 0)=\alpha_{i}(x, t) \leqq u_{i}(x, t) \leqq \beta_{i}(x, t)
$$

for $(x, t) \in \overline{\mathscr{D}} \times[0, T], i=1, \ldots, n$.

Proof Since $u_{i}, \alpha_{i}, \beta_{i} \in H^{2+l, 1+l / 2}(\overline{\mathscr{D}})$, there are constants $K$ and $M$ such that $\left|\alpha_{i}\right| \leqq K$, $\left|\beta_{i}\right| \leqq K,\left|u_{i}\right| \leqq K,\left|\Delta u_{i}\right| \leqq M,\left|\operatorname{grad} u_{i}\right|^{2} \leqq M$ for all $(x, t) \in \overline{\mathscr{D}}_{T}, i=1, \ldots, n$. The assumptions on $f_{i}, a_{i}$ and $\sigma_{i}$ imply that there are constants $R$ and $B$ so that for each $i=1, \ldots, n$, we have $\left|\sigma_{i}^{\prime}(s)\right| \leqq R, \quad\left|\sigma_{i}^{\prime \prime}(s)\right| \leqq R$ for $0 \leqq s \leqq 2 K$, and $\left|a_{i}(x)+f_{i}\left(s_{1}, \ldots, s_{n}\right)\right| \leqq B$ for $x \in \bar{D}$, $0 \leqq s_{i} \leqq 2 K, i=1, \ldots, n$.

Let $0<\varepsilon<K[1+3(B+2 M R+K L n) T]^{-1}$, where $\frac{1}{2} L$ is a bound for the absolute values of all first partial derivatives of $f_{i}\left(s_{1}, \ldots, s_{n}\right), 0 \leqq s_{i} \leqq 2 K, i=1, \ldots, n$. Define, for $(x, t) \in \overline{\mathscr{D}}_{T}$, $i=1, \ldots, n$,

$$
\begin{aligned}
& u_{i}^{+}(x, t)=u_{i}(x, t)+\varepsilon[1+3(B+2 M R+K L n) t] \\
& u_{i}^{-}(x, t)=u_{i}(x, t)-\varepsilon[1+3(B+2 M R+K L n) t] .
\end{aligned}
$$


By hypothesis, we have

$$
\alpha_{i}(x, t)<u_{i}^{+}(x, t) \quad \text { and } \quad u_{i}^{-}(x, t)<\beta_{i}(x, t)
$$

for $x \in \overline{\mathscr{D}}, t=0, i=1, \ldots, n$. Suppose one of these inequalities fails at some point in $\overline{\mathscr{D}} \times\left(0, \tau_{1}\right)$, where $\tau_{1}=\min \{T, 1 /(3(B+2 M R+K L n))\}$; and $\left(x_{1}, t_{1}\right)$ is a point in $\mathscr{\mathscr { D }} \times\left(0, \tau_{1}\right)$ with minimal $t_{1}$ where (2.14) fails. At $\left(x_{1}, t_{1}\right), \sigma_{i}=u_{i}^{+}$or $u_{i}^{-}=\beta_{i}$ for some $i$. Assume the former is the case; a similar proof holds for the latter case.

Suppose further that at $\left(x_{1}, t_{1}\right), \alpha_{j}=u_{j}^{+}$(a simpler proof will work if $\alpha_{i}=u_{i}^{+}$at $\left(x_{1}, t_{1}\right)$ for $i \neq j)$, we consider separately the situations for $x_{1} \in\left(\mathscr{D} \backslash \mathscr{D}^{\prime}\right)$ or $x_{1} \in \mathscr{D}^{\prime}$. If $x_{1} \in \mathscr{D} \backslash \mathscr{D}^{\prime}$, we have $u_{j}^{+}(x, t)>0$ for $t<t_{1}, x \in \bar{D}$ and $u_{j}^{+}\left(x_{1}, t_{1}\right)=0$. Observe that $x_{1} \notin \delta \mathscr{D}$ because $u_{j}^{+}\left(x, t_{1}\right)>u_{j}\left(x, t_{1}\right) \geqq 0$ for $x \in \delta \mathscr{D}$, by (2.11). However, for $(x, t) \in \mathscr{D} \times(0, T]$ :

$$
\begin{aligned}
\frac{\partial}{\partial t}\left(-u_{j}^{+}\right)= & \frac{-\partial}{\partial t}\left(u_{j}\right)-\varepsilon 3(B+2 M R+K L n) \\
= & -\sigma_{j}\left(u_{j}\right) \Delta u_{j}-\sigma_{j}^{\prime}\left(u_{j}\right)\left|\operatorname{grad} u_{j}\right|^{2}-u_{j}\left[a_{j}(x)+f_{j}\left(u_{1}, \ldots, u_{n}\right)\right] \\
& -\varepsilon 3(B+2 M R+K L n) \\
= & -\sigma_{j}\left(u_{j}^{+}\right) \Delta u_{j}+\left[\sigma_{j}\left(u_{j}^{+}\right)-\sigma_{j}\left(u_{j}\right)\right] \Delta u_{j} \\
& -\sigma_{j}^{\prime}\left(u_{j}\right)\left|\operatorname{grad} u_{j}\right|^{2}+\left[u_{j}^{+}-u_{j}\right]\left[a_{j}+f_{j}\left(u_{1}, \ldots, u_{n}\right)\right] \\
& -u_{j}^{+}\left[a_{j}+f_{j}\left(u_{1}, \ldots, u_{n}\right)\right]-\varepsilon 3(B+2 M R+K L n) .
\end{aligned}
$$

Recalling that $\sigma_{j}\left(u_{j}^{+}\right)>0$; and at $\left(x_{1}, t_{1}\right)$ we have $\operatorname{grad} u_{j}=\operatorname{grad} u_{j}^{+}=0, \Delta u_{j}=\Delta u_{j}^{+} \geqq 0$, (2.15) implies that

$$
\begin{aligned}
\left.\frac{\partial}{\partial t}\left(-u_{j}^{+}\right)\right|_{\left(x_{1}, t_{1}\right)} \leqq & R \varepsilon\left[1+3(B+2 M R+K L n) t_{1}\right] M+\varepsilon\left[1+3(B+2 M R+K L n) t_{1}\right] B \\
& -\varepsilon 3(B+2 M R+K L n) \\
\leqq & M R \varepsilon 2+B \varepsilon 2-\varepsilon 3[B+2 M R+K L n]<0
\end{aligned}
$$

contradicting the definition of $\left(x_{1}, t_{1}\right)$.

If $x_{1} \in \mathscr{D}^{\prime}$, we have $u_{j}^{+}(x, t)>\alpha_{j}(x, t)$ for $t<t_{1}, x \in \overline{\mathscr{D}}$; and $u_{j}^{+}\left(x_{1}, t_{1}\right)=\alpha_{j}\left(x_{1}, t_{1}\right)=\delta \theta\left(x_{1}\right)$. But for $(x, t) \in \mathscr{D}^{\prime} \times(0, T]$

$$
\begin{aligned}
\frac{\partial}{\partial t}\left(\alpha_{j}-u_{j}^{+}\right) \leqq & \operatorname{div}\left(\sigma_{j}\left(\alpha_{j}\right) \nabla \alpha_{j}\right)+\alpha_{j}\left[a_{j}+f_{j}\left(\beta_{1}, \ldots, \beta_{j-1}, \alpha_{j}, \beta_{j+1}, \ldots, \beta_{n}\right)\right] \\
& -\operatorname{div}\left(\sigma_{j}\left(u_{j}\right) \nabla u_{j}\right)-u_{j}\left[a_{j}+f_{j}\left(u_{1}, \ldots, u_{n}\right)\right]-\varepsilon 3(B+2 M R+K L n)
\end{aligned}
$$




$$
\begin{aligned}
= & \sigma_{j}\left(\alpha_{j}\right) \Delta \alpha_{j}+\sigma_{j}^{\prime}\left(\alpha_{j}\right)\left|\operatorname{grad} \alpha_{j}\right|^{2}+\alpha_{j}\left[a_{j}+f_{j}\left(\beta_{1}, \ldots, \beta_{j-1}, \alpha_{j}, \beta_{j+1}, \ldots, \beta_{n}\right)\right] \\
& -\sigma_{j}\left(u_{j}^{+}\right) \Delta u_{j}+\left[\sigma_{j}\left(u_{j}^{+}\right)-\sigma_{j}\left(u_{j}\right)\right] \Delta u_{j}+\left[\sigma_{j}^{\prime}\left(u_{j}^{+}\right)-\sigma_{j}^{\prime}\left(u_{j}\right)\right]\left|\operatorname{grad} u_{j}\right|^{2} \\
& -\sigma_{j}^{\prime}\left(u_{j}^{+}\right)\left|\operatorname{grad} u_{j}\right|^{2}+\left(u_{j}^{+}-u_{j}\right)\left[a_{j}+f_{j}\left(u_{1}, \ldots, u_{n}\right)\right]-u_{j}^{+}\left[a_{j}+f_{j}\left(u_{1}, \ldots, u_{n}\right)\right] \\
& -\varepsilon 3(B+2 M R+K L n) .
\end{aligned}
$$

At $\left(x_{1}, t_{1}\right)$, we have $u_{j}^{+}=\alpha_{j}$, grad $u_{j}=\operatorname{grad} u_{j}^{+}=\operatorname{grad} \alpha_{j}, \Delta\left(\alpha_{j}-u_{j}\right)=\Delta\left(\alpha_{j}-u_{j}^{+}\right) \leqq 0$, thus (2.17) gives

$$
\begin{aligned}
\left.\frac{\partial}{\partial t}\left(\alpha_{j}-u_{j}^{+}\right)\right|_{\left(x_{1}, t_{1}\right)} & =\sigma_{j}\left(\alpha_{j}\right) \Delta\left(\alpha_{j}-u_{j}\right)+\alpha_{j}\left[f_{j}\left(\beta_{1}, \ldots, \beta_{j-1}, \alpha_{j}, \beta_{j+1}, \ldots, \beta_{n}\right)-f_{j}\left(u_{1}, \ldots, u_{n}\right)\right] \\
& +\left[\sigma_{j}\left(u_{j}^{+}\right)-\sigma_{j}\left(u_{j}\right)\right] \Delta u_{j}+\left[\sigma_{j}^{\prime}\left(u_{j}^{+}\right)-\sigma_{j}^{\prime}\left(u_{j}\right)\right]\left|\operatorname{grad} u_{j}\right|^{2} \\
& +\left(u_{j}^{+}-u_{j}\right)\left[a_{j}+f_{j}\left(u_{1}, \ldots, u_{n}\right)\right]-\varepsilon 3(B+2 M R+K L n) .
\end{aligned}
$$

Moreover, at $\left(x_{1}, t_{1}\right)$ we have

$$
\begin{aligned}
& f_{j}\left(\beta_{1}, \ldots, \beta_{j-1}, \alpha_{j}, \beta_{j+1}, \ldots, \beta_{n}\right)-f_{j}\left(u_{1}, \ldots, u_{n}\right) \\
& \quad \leqq f_{j}\left(\tilde{u}_{1}^{-}, \ldots, \tilde{u}_{j-1}^{-}, u_{j}^{+}, \tilde{u}_{j+1}^{-}, \ldots, \tilde{u}_{n}^{-}\right)-f_{j}\left(u_{1}, \ldots, u_{n}\right) \leqq L \varepsilon\left[1+3(B+2 M R+K L n) t_{1}\right] n
\end{aligned}
$$

where $\tilde{u}_{i}^{-}\left(x_{1}, t_{1}\right)=\max \left\{u_{i}^{-}\left(x_{1}, t_{1}\right), \alpha_{i}\left(x_{1}, t_{1}\right)\right\}$, because $\left|u_{i}-\tilde{u}_{i}^{-}\right| \leqq\left|u_{i}^{+}-u_{i}^{-}\right|$. Consequently, (2.18) gives

$$
\begin{aligned}
\left.\frac{\partial}{\partial t}\left(\alpha_{j}-u_{j}^{+}\right)\right|_{\left(x_{1}, t_{1}\right)} \leqq & K L n \varepsilon\left[1+3(B+2 M R+K L n) t_{1}\right]+2 M R \varepsilon\left[1+3(B+2 M R+K L n) t_{1}\right] \\
& +B \varepsilon\left[1+3(B+2 M R+K L n) t_{1}\right]-\varepsilon 3(B+2 M R+K L n) \\
\leqq & K L n \varepsilon 2+4 M R \varepsilon+2 B \varepsilon-\varepsilon 3(B+2 M R+K L n)<0
\end{aligned}
$$

contradicting the definition of $\left(x_{1}, t_{1}\right)$. From these contradictions, we conclude that $u_{j}^{+}(x, t)>\alpha_{j}(x, t)$ for $(x, t) \in \bar{D} \times\left[0, \tau_{1}\right)$. Passing to the limit as $\varepsilon \rightarrow 0^{+}$, we obtain $u_{j}(x, t) \geqq \alpha_{j}(x, t)$ in $\overline{\mathscr{D}} \times\left[0, \tau_{1}\right]$.

If at $\left(x_{1}, t_{1}\right)$, we have $\alpha_{m}=u_{m}^{+}$for $m \neq \mathrm{j}$, then $u_{m}^{+}(x, t)>0$ for $t<t_{1}, x \in \bar{D}$ and $u_{m}^{+}\left(x_{1}, t_{1}\right)=0$, with $x_{1} \notin \delta \mathscr{D}$. For $x_{1} \in \mathscr{D}$, repeat the arguments in (2.15) to (2.16), with $j$ replaced by $m$. (There is no need for arguments analogous to (2.17) to (2.20)). We obtain $u_{m}^{+}>\alpha_{m}=0$ for $(x, t) \in \mathscr{D} \times\left[0, \tau_{1}\right)$, and consequently $u_{m} \geqq \alpha_{m}=0$ for $(x, t) \in \overline{\mathscr{D}} \times\left[0, \tau_{1}\right]$.

If at $\left(x_{1}, t_{1}\right), u_{i}^{-}=\beta_{i}$ for some $i$, we show that

$$
\left.\frac{\partial}{\partial t}\left(\beta_{i}-u_{i}^{-}\right)\right|_{\left(x_{1}, t_{1}\right)}>0
$$


by means of (2.10), in a way similar to the arguments that led to (2.17) to (2.20), but with inequalities reversed. Passing to the limit as $\varepsilon \rightarrow 0^{+}$, we again obtain $u_{i} \leqq \beta_{i}$ for $(x, t) \in \overline{\mathscr{D}} \times\left[0, \tau_{1}\right]$.

If $\tau_{1}<T$, we repeat the above arguments by starting to define $u_{i}^{+}, u_{i}^{-}$with (2.13), with $t$ in the square brackets on the right side of the formulas, replaced by $\left(t-\tau_{1}\right)$. This leads to $\alpha_{i} \leqq u_{i} \leqq \beta_{i}$ for $x \in \overline{\mathscr{D}}, \tau_{1} \leqq t \leqq \min \{T, 2 / 3(B+2 M R+K L n)\}$ etc. Eventually, we obtain (2.12) in $\overline{\mathscr{D}} \times[0, T]$.

Remark. The assumption $\sigma_{i}^{\prime}(s) \geqq 0, i=1, \ldots, n$, has never been used in the proof of Theorem 2.2. However, $\sigma_{k}^{\prime} \geqq 0$ is essential for establishing the positivity of expression (2.8), in the proof of Theorem 2.1 .

The following is an immediate consequence of Theorem 2.1. It gives a sufficient condition for the coexistence of $r$ species, $0<r \leqq n$, in $\mathscr{D}$.

Theorem 2.3. Let $b_{i} \geqq\left|r_{i}^{-1}\right| \max \left\{a_{i}(x) \mid x \in \overline{\mathscr{D}}\right\}, i=1, \ldots, n$. Suppose there exist $r$ subdomains $\mathscr{D}_{k_{1}}, \ldots, \mathscr{D}_{k_{r}}\left(0<r \leqq n, k_{1}, \ldots, k_{r}\right.$ are distinct positive integers $\left.\leqq n\right)$ in $\mathscr{D}$, with the property that:

$$
a_{k_{i}}(x)-\sigma_{k_{i}}(0) \lambda_{k_{i}}+f_{k_{i}}\left(b_{1}, \ldots, b_{k_{i}-1}, 0, b_{k_{i}+1}, \ldots, b_{n}\right)>0
$$

for $x \in \mathscr{D}_{k_{i}}, i=1, \ldots, r$. (Here, $\lambda=\lambda_{k_{i}}>0$ is the first eigenvalue for the problem: $\Delta \phi+\lambda \phi=0$ in $\mathscr{D}_{k_{i}}, \phi=0$ on $\left.\delta \mathscr{D}_{k_{i}}\right)$. Let $\left(u_{1}, \ldots, u_{n}\right)$ be a solution of (1.1) with each component in $H^{2+l, 1+l / 2}\left(\overline{\mathscr{D}}_{T}\right), T>0$; and assume initially that

$$
\begin{gathered}
0 \leqq u_{i}(x, 0) \leqq b_{i}, \quad x \in \overline{\mathscr{D}}, \quad i=1, \ldots, n \\
0<u_{k_{i}}(x, 0), \quad x \in \overline{\mathscr{D}}_{k_{i}}, \quad i=1, \ldots, r
\end{gathered}
$$

Then the solution satisfies

$$
0<u_{k_{i}}(x, t), \quad(x, t) \in \overline{\mathscr{D}}_{k_{i}} \times[0, T], \quad i=1, \ldots, r
$$

Moreover $u_{k_{i}}(x, t) \geqq \delta>0$ for all $x$ in any compact set contained in $\mathscr{D}_{\boldsymbol{k}_{i}}, 0 \leqq t \leqq T$ (where $\delta$ is some constant depending on the compact set, independent of $T)$; and

$$
0 \leqq u_{i}(x, t) \leqq b_{i} \quad(x, t) \in \overline{\mathscr{D}}_{T}
$$

Note that the $k_{i}$ th species will have, for all time under consideration, its concentration bounded below by positive constants in compact subsets of $\mathscr{D}_{k_{i}}$. The simplest situation happens when $\mathscr{D}_{k_{1}}=\mathscr{D}_{k_{2}} \ldots=\mathscr{D}_{k_{r}}$; otherwise, the different species will primarily survive at different subregions in $\mathscr{D}$.

\section{Criteria for extinction of the $k$ th species ( $u_{k}$ tending to zero)}

In this section we consider the initial boundary value problem:

$$
\frac{\partial u_{i}}{\partial t}=\operatorname{div}\left(\sigma_{i}\left(u_{i}\right) \nabla u_{i}\right)+u_{i}\left[a_{i}(x)+f_{i}\left(u_{1}, \ldots, u_{n}\right)\right] \quad \text { in } \mathscr{D} \times(0, T], \quad i=1, \ldots, n
$$




$$
\begin{gathered}
u_{i}(x, 0)=\phi_{i}(x) \geqq 0, \quad x \in \overline{\mathscr{D}}, \quad i=1, \ldots, n \\
u_{i}(x, t)=\Phi_{i}(x) \geqq 0, \quad(x, t) \in \delta \mathscr{D} \times[0, T], \quad i=1, \ldots, n
\end{gathered}
$$

with the special homogeneous boundary condition on the $k$ th component:

$$
\Phi_{k}(x) \equiv 0 \quad x \in \delta \mathscr{D}
$$

We assume all the hypotheses described in Section 1 (i.e. (1.2) to (1.5) etc). Moreover, we assume $\phi_{i}(x)=\Phi_{i}(x)$ if $x \in \delta \mathscr{D}, \phi_{i} \in H^{2+l}(\overline{\mathscr{D}})$, and

$$
\left.\left\{\operatorname{div}\left(\sigma_{i}\left(\phi_{i}\right) \nabla \phi_{i}\right)+\phi_{i}\left[a_{i}(x)+f_{i}\left(\phi_{1}, \ldots, \phi_{n}\right)\right]\right\}\right|_{x \in \delta \mathscr{D}}=0 \quad \text { for } \quad i=1, \ldots, n .
$$

The following theorem describes a sufficient condition for the decay of the $k$ th species.

Theorem 3.1. Suppose that

$$
a_{k}(x)<\sigma_{k}(0) \lambda^{1} \quad \text { for all } x \in \overline{\mathscr{T}}
$$

(where $\lambda=\lambda^{1}$ is the first eigenvalue of $\Delta w+i w=0$ in $/, w=0$ on $\delta \mathscr{D}$ ). Let $C_{i}>0$, $i=1, \ldots, n$ be such that for $x \in \overline{\mathscr{D}}$

$$
a_{i}(x)+f_{i}\left(0, \ldots, 0, C_{i}, 0, \ldots, 0\right) \leqq 0
$$

(here $C_{i}$ appears in the ith component), and $\bar{C}_{i}=\max \left\{C_{i}, \sup _{x \in \bar{g}} \phi_{i}(x)\right\}$. Then there is $a$ constant $q>0$ so that the property:

$$
\sigma_{k}^{\prime}(s) \leqq q, \text { for all } 0 \leqq s \leqq \bar{C}_{k}
$$

implies that any solution $\left(u_{1}, \ldots, u_{n}\right)$ of (3.1) to (3.3) with each component in $H^{2+l, 1+l / 2}\left(\overline{\mathscr{D}}_{T}\right), T>0$ must satisfy:

$$
0 \leqq u_{k}(x, t) \leqq K e^{-\varepsilon t} \quad \text { in } \quad \overline{\mathscr{D}}_{T}
$$

where $K, \varepsilon$ are positive constants independent of $T$. Moreover, we have

$$
0 \leqq u_{i}(x, t) \leqq \bar{C}_{i}, \quad i=1, \ldots, n \quad \text { in } \quad \overline{\mathscr{D}}_{T}
$$

Remarks. $\quad C_{i}$ exist by hypothesis (1.3); the size of $q$ is given in (3.17) in terms of the principle eigenfunction of a domain $\hat{\mathscr{D}} \supset \overline{\mathscr{D}}$.

Proof. Define $\tilde{a}(x, w)$ for $x \in \overline{\mathscr{D}} \times R$ by

$$
\tilde{a}(x, w)= \begin{cases}w\left[a_{k}(x)+f_{k}(0, \ldots, 0, w, 0, \ldots, 0)\right] & \text { if } \quad(x, w) \in \overline{\mathscr{D}} \times\left[-\bar{C}_{k}, \bar{C}_{k}\right] \\ h(w)\left[a_{k}(x)+f_{k}(0, \ldots, 0, h(w), 0, \ldots, 0)\right] & \text { if } \quad x \in \overline{\mathscr{D}}, w \geqq C_{k} \\ -h(-w)\left[a_{k}(x)+f_{k}(0, \ldots, 0,-h(-w), 0, \ldots, 0)\right. & \text { if } \quad x \in \overline{\mathscr{D}}, w \leqq-\bar{C}_{k}\end{cases}
$$


where $h(w)=2 \bar{C}_{k}-\bar{C}_{k} \exp \left\{\bar{C}_{k}^{-1}\left(\bar{C}_{k}-w\right)\right\}$, with $w, \pm h(w)$ appearing on the $k$ th component of $f_{k}$. Define

$$
\tilde{\sigma}(w)=\left\{\begin{array}{lll}
\sigma_{k}(w) & \text { if } \quad w \geqq 0 \\
\delta+\left(\sigma_{k}(0)-\delta\right) \exp \left\{\sigma_{k}^{\prime}(0)\left[\alpha_{k}(0)-\delta\right]^{-1} w\right\} & \text { if } \quad w<0
\end{array}\right.
$$

where $0<\delta<\sigma_{k}(0)$. (Note that $\tilde{\sigma}^{\prime}(w)$ is uniformly Hölder continuous with exponent $l$ in any bounded interval in $R)$. Let $w=w_{k}(x, t)$ be a solution in $H^{2+l}\left(\overline{\mathscr{D}}_{T}\right)$ for the initial boundary value problem:

$$
\begin{array}{r}
\frac{\partial w}{\partial t}=\operatorname{div}(\tilde{\sigma}(w) \operatorname{grad} w)+\tilde{a}(x, w) \quad \text { in } \mathscr{D} \times(0, T] \\
w(x, 0)=u_{k}(x, 0), \quad x \in \overline{\mathscr{D}} \\
w(x, t)=0, \quad(x, t) \in \delta \mathscr{D} \times[0, T]
\end{array}
$$

(By [4], Theorem 6.1, p. 452, (3.9) possesses a unique solution in $H^{2+l},{ }^{1+l / 2}\left(\overline{\mathscr{D}}_{T}\right)$. Note that $a_{i}, a, u, p_{i}$ in [4] correspond respectively to $\tilde{\sigma}(w) w_{x_{i}},-\tilde{a}(x, w), w, w_{x_{i}}$ in (3.9). Also $\partial \tilde{a} / \partial w$ exist in $\overline{\mathscr{D}} \times \mathbb{R}$; and $\tilde{a}, \partial \tilde{a} / \partial w$ are bounded. Conditions (a) to (f) in Theorem 6.1 in [4] are all satisfied.)

The function $\beta(x) \equiv \bar{C}_{k}$ satisfies:

$$
\operatorname{div}\left(\sigma_{k}(\beta) \nabla \beta\right)+\beta\left[a_{k}(x)+f_{k}(0, \ldots, 0, \beta, 0, \ldots, 0)\right]-\frac{\partial \beta}{\partial t} \leqq 0
$$

by (3.5) and (1.2); while the function $\alpha(x) \equiv 0$ satisfies:

$$
\operatorname{div}\left(\sigma_{k}(\alpha) \nabla \alpha\right)+\alpha\left[a_{k}(x)+f_{k}(0, \ldots, 0, \alpha, 0, \ldots, 0)\right]-\frac{\partial \alpha}{\partial t} \geqq 0
$$

As long as $0 \leqq w \leqq \bar{C}_{k}$, equation (3.9) is the same as

$$
\operatorname{div}\left(\sigma_{k}(w) \nabla w\right)+w\left[a_{k}(x)+f_{k}(0, \ldots, 0, w, 0, . ., 0)\right]-\frac{\partial w}{\partial t}=0
$$

Since $\alpha(x) \leqq w_{k}(x, 0) \leqq \beta(x), x \in \overline{\mathscr{D}}$ and $\alpha(x) \leqq w_{k}(x, t) \leqq \beta(x)$ for $(x, t) \in \delta \mathscr{D} \times[0, T]$, by a variant of Theorem 2.2 , we have

$$
0 \equiv \alpha(x) \leqq w_{k}(x, t) \leqq \beta(x) \equiv \bar{C}_{k} \quad \text { for } \quad(x, t) \in \overline{\mathscr{D}} \times[0, T]
$$

(Note that the proof of (3.13) is simpler than that of Theorem 2.2, and is completely analogous. In proving Theorem 2.2 we essentially used the fact that $f_{m}$ is nonincreasing in its dependence on the $n$th variable $n \neq m$, cf. (2.19). However in (3.12), there is simply one equation with one unknown; therefore the details will be omitted.) 
We next prove that $w_{k}(x, t) \rightarrow 0$ as $t \rightarrow \infty$. Let $\hat{\mathscr{D}} \supset \overline{\mathscr{D}}$ be a domain with its corresponding principle eigenvalue $\lambda=\hat{\lambda}$ for the problem $\Delta \theta+\lambda \theta=0$ in $\hat{\mathscr{D}}, \theta=0$ in $\delta \hat{\mathscr{D}}$, satisfying

$$
a_{k}(x)<\sigma_{k}(0) \hat{\lambda}<\sigma_{k}(0) \lambda^{1}
$$

for $x \in \overline{\mathscr{D}}$. Let $\theta=\psi$ be a corresponding principle eigenfunction. We have $\psi(x)>0$ in $\overline{\mathscr{D}}$. Set $w_{k}(x, t)=z(x, t) \psi(x) e^{-\varepsilon t}$ for $(x, t) \in \bar{D} \times[0, T] \quad(\varepsilon>0$ will be determined later $)$. Substituting into (3.12), multiplying by $e^{\varepsilon t} \psi^{-1}$ and regrouping terms, we obtain:

$$
\begin{aligned}
& \sigma_{k}\left(w_{k}\right) \Delta z+\left[\psi^{-1} \sigma_{k}\left(w_{k}\right) 2(\operatorname{grad} \psi)+\sigma_{k}^{\prime}\left(w_{k}\right) e^{-\varepsilon t} z 2(\operatorname{grad} \psi)\right. \\
& \left.\quad+\sigma_{k}^{\prime}\left(w_{k}\right) e^{-\varepsilon t} \psi(\operatorname{grad} z)\right] \cdot(\operatorname{grad} z)+z\left[\psi^{-1} \sigma_{k}\left(w_{k}\right) \Delta \psi+\sigma_{k}^{\prime}\left(w_{k}\right) e^{-\varepsilon t} \psi^{-1} z|\operatorname{grad} \psi|^{2}\right. \\
& \left.\quad+a_{k}(x)+f_{k}\left(0, \ldots, 0, w_{k}, 0, \ldots, 0\right)+\varepsilon\right]-\frac{\partial z}{\partial t}=0
\end{aligned}
$$

in $\mathscr{D} \times(0, T]$. The coefficient of $z$ in $(3.15)$ can be rewritten as

$$
a_{k}(x)-\hat{\lambda} \sigma_{k}\left(w_{k}\right)+\varepsilon+f_{k}\left(0, \ldots, 0, w_{k}, 0, \ldots, 0\right)+\sigma_{k}^{\prime}\left(w_{k}\right) w_{k}|\operatorname{grad} \psi|^{2} \psi^{-2}
$$

Since $\sigma_{k}^{\prime}(s) \geqq 0$ for $s \geqq 0,(3.14)$ implies that we can choose $\varepsilon>0$ sufficiently small so that $a_{k}(x)-\hat{\lambda} \sigma_{k}\left(w_{k}\right)+\varepsilon<0$ for $(x, t) \in \overline{\mathscr{D}} \times[0, T]$. The last two terms in (3.16) can be written as

$$
\left\{\int_{0}^{1} \frac{\partial f_{k}}{\partial u_{k}}\left(0, \ldots, s w_{k}, 0, \ldots, 0\right) d s+\sigma_{k}^{\prime}\left(w_{k}\right)|\operatorname{grad} \psi|^{2} \psi^{-2}\right\} w_{k} .
$$

By hypothesis (1.3)

$$
\int_{0}^{1} \frac{\partial f_{k}}{\partial u_{k}}\left(0, \ldots, s w_{k}, 0, \ldots, 0\right) d s<r_{k} \quad \text { for all } \quad w_{k} \geqq 0 .
$$

For hypothesis (3.6), we let

$$
q=\left|r_{k}\right|\left[\max _{x \in \overline{\mathscr{D}}}\left\{|\operatorname{grad} \psi|^{2} \cdot \psi^{-2}(x)\right\}\right]^{-1}
$$

Clearly, we then have (3.16) negative for all $0 \leqq w_{k} \leqq \bar{C}_{k}$, from hypothesis (3.6). Since $z(x, t)=0$ on $\delta \mathscr{D} \times[0, T]$, the maximum principle and $(3.15)$ implies that

$$
|z(x, t)| \leqq \max _{x \in \overline{\mathscr{D}}}\left\{u_{k}(x, 0) \psi^{-2}(x)\right\} \quad \text { for } \quad(x, t) \in \mathscr{D} \times[0, T]
$$

We therefore have

$$
\left|w_{k}(x, t)\right| \leqq K e^{-s t} \text { for }(x, t) \in \bar{\zeta} \times[0, T]
$$

where the positive constants $K$ and : are independent of $T$. 
Finally, define $v_{i} \equiv 0$ for $i=1, \ldots, n$ and $w_{i} \equiv \bar{C}_{i}$ for $i \neq k$. Then $v_{i}, w_{i}$ satisfies (2.9), (2.10) for $(x, t) \in \mathscr{D} \times(0, T]$, and (2.11) for $(x, t) \in(\bar{D} \times\{0\}) \cup(\delta \mathscr{D} \times[0, T])$, (with $v_{i}$, $w_{i}$ replacing $\alpha_{i}, \beta_{i}$ respectively.) By a proof completely analogous to that in Theorem 2.2, we conclude that

$$
0=v_{i} \leqq u_{i}(x, t) \leqq w_{i}=\bar{C}_{i} \text { in } \quad \bar{D}_{T}
$$

for $i=1, \ldots, n$. Moreover $u_{k}(x, t) \leqq w_{k}(x, t)$ and (3.18) imply that (3.7) is valid. (Note that the proof of (3.19) is simpler than that in Theorem 2.2, because all $v_{i} \equiv 0$; and to show that $v_{i} \leqq u_{i}$ we need only arguments corresponding to (2.15) and (2.16). There is no need for arguments corresponding to (2.17) and (2.20)).

\section{Remarks on existence}

We now finally give detailed conditions and proof for the existence of a solution to the initial-boundary value problem (1.1). Theorem 4.1 justifies that the solutions in $H^{2+l}\left(\overline{\mathscr{D}}_{T}\right)$, assumed in Sections 2 and 3 , do indeed exist.

Theorem 4.1. Let $\mathscr{D}, f_{i}, a_{i}, \sigma_{i}, i=1, \ldots, n$ satisfy all the conditions as described in Section 1. Let the initial boundary functions $\phi_{i}, \Phi_{i}$ satisfy: $\phi_{i}(x)=\Phi_{i}(x)$ for $x \in \delta \mathscr{D}$, $\phi_{i}(x) \geqq 0$ in $\overline{\mathscr{D}}, \phi_{i}$ has all third partial derivatives continuous in $\overline{\mathscr{D}}$, and

$$
\left\{\operatorname{div}\left(\sigma_{i}\left(\phi_{i}\right) \nabla \phi_{i}\right)+\left.\phi_{i}\left[a_{i}(x)+f_{i}\left(\phi_{1}(x), \ldots, \phi_{n}(x)\right]\right\}\right|_{x \in \delta \mathscr{Q}}=0\right.
$$

for $i=1, \ldots, n$. Then, for any $T>0$, in the class of functions in $H^{2+l, 1+l / 2}\left(\overline{\mathscr{D}}_{T}\right)$, there exists a unique solution for the initial boundary value problem (1.1).

Proof. Let $d_{i}$ be positive numbers satisfying:

$$
\begin{gathered}
d_{i} \geqq\left|r_{i}^{-1}\right| \max \left\{a_{i}(x) \mid x \in \overline{T_{i}}, \quad\right. \text { and } \\
0 \leqq \phi_{i}(x) \leqq d_{i}, \quad x \in \overline{\mathbb{X}}
\end{gathered}
$$

for $i=1, \ldots, n$. Define $c_{i}\left(x, u_{1}, \ldots, u_{n}\right), i=1, \ldots, n,\left(x, u_{1}, \ldots, u_{n}\right) \in \overline{\mathscr{D}} \times \mathbb{R}^{n}$ by:

$$
c_{i}\left(x, u_{1}, \ldots, u_{n}\right)=h_{i}\left(u_{i}\right)\left[a_{i}(x)+f_{i}\left(h_{1}\left(u_{1}\right), \ldots, h_{n}\left(u_{n}\right)\right)\right]
$$

where $h_{i}(s)=\left\{\begin{array}{lll}s & \text { if } & |s| \leqq d_{i} \\ \rho_{i}(s) & \text { if } & |s|>d_{i}\end{array}\right.$

with $\rho_{i}(s)$ a twice continuously differentiable function for $|s| \geqq d_{i}$, and $\left|\rho_{i}(s)\right| \leqq 2 d_{i}$, $\rho_{i}\left( \pm d_{i}\right)= \pm d_{i}, \rho_{i}^{\prime}\left( \pm d_{i}\right)=1$, and $\rho_{i}^{\prime \prime}\left(d_{i}\right)=0$. Extend $\tilde{\sigma}_{i}(s)$ positively to $(-\infty, 0)$ by letting $\tilde{\sigma}_{i}(s)=\sigma_{i}(s)$ for $s \in[0, \infty)$, with $\tilde{\sigma}_{i}(s)$ twice continuously differentiable for $s \in(-\infty, \infty)$, and $\tilde{\sigma}_{i}(s) \geqq\left(\sigma_{i}(0) / 2\right)>0$ for $s \in(-\infty, 0), i=1, \ldots, n$. 
We consider the initial boundary value problem:

$$
\begin{aligned}
\frac{\partial z_{i}}{\partial t}(x, t)= & \tilde{\sigma}_{i}\left(h_{i}\left(z_{i}+\phi_{i}(x)\right)\right) \Delta z_{i}+\tilde{\sigma}_{i}^{\prime}\left(h_{i}\left(z_{i}+\phi_{i}\right)\right) \sum_{j=1}^{n}\left[\left(z_{i}\right)_{x_{j}}+2\left(\phi_{i}\right)_{x_{j}}\right] \cdot\left(z_{i}\right)_{x_{j}} \\
& +\tilde{\sigma}_{i}^{\prime}\left(h_{i}\left(z_{i}+\phi_{i}\right)\right) \sum_{j=1}^{n}\left(\phi_{i}\right)_{x_{j}}^{2}+\tilde{\sigma}_{i}\left(h_{i}\left(z_{i}+\phi_{i}\right)\right) \Delta \phi_{i}+c_{i}\left(x, z_{1}+\phi_{1}, \ldots, z_{n}+\phi_{n}\right)
\end{aligned}
$$

for $(x, t) \in \mathscr{D} \times(0, T], i=1, \ldots, n$;

$$
z_{i}(x, 0)=0 \text { in } \overline{\mathscr{D}} \text { and } z_{i}(x, t)=0 \text { for }(x, t) \in \delta \mathscr{D} \times[0, T]
$$

(Note that if we let $u_{i}(x, t)=z_{i}(x, t)+\phi_{i}(x)$, and if $0 \leqq u_{i}(x, t) \leqq d_{i}, i=1, \ldots, n$, then $u_{i}(x, t)$ satisfies:

$$
\frac{\partial u_{i}}{\partial t}=\sigma_{i}\left(u_{i}\right) \Delta u_{i}+\sigma_{i}^{\prime}\left(u_{i}\right)\left|\operatorname{grad} u_{i}\right|^{2}+u_{i}\left[a_{i}(x)+f_{i}\left(u_{1}, \ldots, u_{n}\right)\right]
$$

Moreover, $u_{i}$ satisfies the initial boundary conditions of (1.1)). Apply Theorem 7.1 on $\mathrm{p}$. 596 of [4]. The positivity of $\tilde{\sigma}_{i}$ and the boundedness of the last three terms of (4.2) imply that condition (a) in Theorem 7.1 is satisfied. (6.3) of (b) in [4] is satisfied by letting $P(|p|,|u|)=C(1+|p|)^{-2}$ for some large constant $C$ and $\varepsilon(|u|)=0$. The smoothness of $\phi_{i}, \tilde{\sigma}_{i}$ and $h_{i}$ ensure that (c) is satisfied. Compatibility condition (4.1) gives (d). Consequently, Theorem 7.1 , p. 596 in [4], gives a unique solution $z=\left(z_{1}(x, t), \ldots, z_{n}(x, t)\right)$ to (4.2), (4.3) for $(x, t) \in \overline{\mathscr{D}} \times[0, T]$, in the class $H^{2+l, 1+l / 2}\left(\overline{\mathscr{D}}_{T}\right)$.

We next show that $0 \leqq z_{i}(x, t)+\phi_{i}(x) \leqq d_{i}, i=1, \ldots, n$. Let $\hat{\alpha}_{i}(x, t) \equiv 0$ and $\hat{\beta}_{i}(x, t) \equiv d_{i}$, $i=1, \ldots, n$. Each function $\hat{\alpha}_{i}$ satisfies $(2.9)$ in $\mathscr{D} \times(0, T]$ (with $\hat{\alpha}_{i}, \hat{\beta}_{i}$ replacing $\alpha_{i}, \beta_{i}$ respectively). Each function $\hat{\beta}_{i}$ satisfies:

$$
\begin{aligned}
\operatorname{div} & \left(\sigma_{i}\left(\hat{\beta}_{i}\right) \nabla \hat{\beta}_{i}\right)+\hat{\beta}_{i}\left[a_{i}(x)+f_{i}\left(\hat{\alpha}_{1}, \ldots, \hat{\alpha}_{i-1}, \hat{\beta}_{i}, \hat{\alpha}_{i+1}, \ldots, \hat{\alpha}_{n}\right)\right]-\frac{\partial \hat{\beta}_{i}}{\partial t} \\
& =d_{i}\left[a_{i}(x)+f_{i}\left(0, \ldots, 0, d_{i}, 0, \ldots, 0\right)\right] \\
& =d_{i}\left[a_{i}(x)+\int_{0}^{d_{i}} \frac{\partial f_{i}}{\partial s_{i}}\left(0, \ldots, 0, s_{i}, 0, \ldots, 0\right) d s_{i}\right] \\
& \leqq d_{i}\left[a_{i}(x)+r_{i} d_{i}\right] \leqq d_{i}\left[a_{i}(x)-\max \left\{a_{i}(x) \mid x \in \overline{\mathscr{D}}\right\}\right] \leqq 0
\end{aligned}
$$

in $\mathscr{D} \times(0, T]$. For $i=1, \ldots, n,(x, t) \in \overline{\mathscr{D}} \times[0, T]$, let

$$
u_{i}(x, t)=z_{i}(x, t)+\phi_{i}(x)
$$

The function $u_{i}$ satisfies (4.4) for $x \in \mathscr{D}, 0<t \leqq t_{1} \leqq T$ as long as $\hat{\alpha}_{i} \leqq u_{i}(x, t) \leqq \hat{\beta}_{i}$ for $(x, t) \in \overline{\mathscr{D}} \times\left[0, t_{1}\right]$. By arguments exactly as given in Theorem 2.2 , we can show that 
$\hat{\alpha}_{i} \leqq u_{i} \leqq \hat{\beta}_{i}$ for all $(x, t) \in \overline{\mathscr{D}} \times[0, T], i=1, \ldots, n$. (Note that our present situation is even simpler because all $\hat{\alpha}_{i} \equiv 0$, and we need only those arguments from (2.13) to (2.16). Those arguments from (2.17) to (2.20) for the $u_{j} \geqq \alpha_{j}$ case will not be necessary). The a-priori bound, $\hat{\alpha}_{i} \leqq u_{i} \leqq \hat{\beta}_{i}$ in $\overline{\mathscr{D}} \times[0, T]$, consequently implies that $u(x, t)$ is the unique solution of the initial value problem (1.1), in $H^{2+l, 1+l / 2}\left(\overline{\mathscr{D}}_{T}\right)$.

Remark 4.1. The above theorem shows that the solution $u(x, t)$ exists in $\overline{\mathscr{D}}_{T}$ for all $T>0$. Under the assumptions of Theorem 3.1 and Theorem 4.1, we therefore have $u_{k}(x, t) \rightarrow 0$ uniformly for $x \in \overline{\mathscr{D}}$, as $t \rightarrow+\infty$.

\section{REFERENCES}

1. P. Brown, Decay to uniform states in ecological interactions, SIAM J. Appl. Math. 38 (1980), 22-37.

2. P. FIFE and M. TANG, Comparison principles for reaction-diffusion systems: irregular comparison functions and applications to questions of stability and speed of propagation of disturbances, J. of Diff. Eq. 40 (1981), 168-185.

3. P. Kareiva, Dispersal as a passive diffusion: an analysis of field experiments involving herbivorous insects, Manuscript (1981).

4. O. Ladyżenskaja, V. Solonnikov, and N. Ural'ceva, Linear and quasilinear equations of parabolic type, Transl. of Math. Monograph AMS 23 (1968).

5. A. LEUNG, Equilibria and stabilities for competing-species reaction-diffusion equations with Dirichlet boundary data, J. Math. Anal. Appl. 73 (1980), 204-218.

6. A. Leung, Stabilities for equilibria of competing-species reaction-diffusion equations with homogeneous Dirichlet conditions, Funk. Ekv. (Ser. Interna.) 24 (1981), 201-210.

7. S. Levin, Models of population dispersal, differential equations and applications, Ecology, Epidemics and Population Problems, edited by S. Busenberg and K. Cooke (Academic Press, 1981).

8. M. Mimura, Y. Nishiura and M. Yamaguti, Some diffusive prey and predator systems and their bifurcation problem, Annals of the N.Y. Academy of Sciences 316 (1979), 490-510.

9. P. De Mottoni, A. Schiaffino, and A. Tesei, On stable space dependent stationary solutions of a competition system with diffusion, Reprint, (1981).

10. А. Окиво, Diffusion and Ecological Problems: Mathematical Models (Springer-Verlag, Berlin, 1980).

11. F. Rothe, A nonhomogeneous predator-prey system with patchiness, Preprint, (1981).

12. L. Z $\mathrm{HOU}$ and C. PAO, Asymptotic behavior of a competition-diffusion system in population dynamics, Nonlinear Anal. Th. Meth. and Appl. 6 (1982), 1163-1184.

Department of Mathematical Sciences

UNIVERSITY OF CINCINNATI

Cincinnati, Ohio 45221 